\title{
The Benefit of Object Interactions Arises in the Lateral Occipital Cortex Independent of Attentional Modulation from the Intraparietal Sulcus: A Transcranial Magnetic Stimulation Study
}

\author{
Jiye G. Kim, ${ }^{1}$ Irving Biederman, ${ }^{1,2}$ and Chi-Hung Juan ${ }^{3,4,5}$ \\ 'Department of Psychology and ${ }^{2}$ Neuroscience Program, University of Southern California, Los Angeles, California 90089, ${ }^{3}$ Institute of Cognitive \\ Neuroscience, National Central University, Jhongli 320, Taiwan, ${ }^{4}$ Laboratories for Cognitive Neuroscience, National Yang-Ming University, Taipei 112, \\ Taiwan, and 5Institute of Network Learning Technology, National Central University, Jhongli 320, Taiwan
}

Our visual experience is generally not of isolated objects, but of scenes, where multiple objects are interacting. Such interactions (e.g., a watering can positioned to pour water toward a plant) have been shown to facilitate object identification compared with when the objects are depicted as not interacting (e.g., a watering can positioned away from the plant) (Green and Hummel, 2004, 2006). What is the neural basis for this advantage? Recent fMRI studies have identified the lateral occipital cortex (LO) as a potential neural origin of this behavioral benefit, as LO showed greater responses to object pairs depicted as interacting compared with when they are not (Kim and Biederman, 2010; Roberts and Humphreys, 2010). However, it is possible that LO was modulated by an attention-sensitive region, the intraparietal sulcus (IPS), which sometimes showed a similar pattern of responses as that of LO in the Kim and Biederman (2010) investigation. To test this hypothesis, we delivered transcranial magnetic stimulation (TMS) to human subjects' LO and IPS while they detected a target object that was or was not interacting with another object to form a scene. TMS delivered to LO but not IPS abolished the facilitation in identifying interacting objects compared with noninteracting depictions observed in the absence of TMS, suggesting that it is $\mathrm{LO}$ and not IPS that is critical for the coding of object interactions.

\section{Introduction}

Objects in our environment typically appear in scenes, where they tend to be interacting with other objects. These relations are automatically processed and have been shown to affect the perceptibility of the objects themselves (Biederman et al., 1974, 1982; Green and Hummel, 2006). For example, Green and Hummel (2006) showed that object recognition is facilitated when a pair of objects is depicted as interacting (e.g., a pitcher positioned to be pouring into a glass) to form a scene compared with when the objects are not interacting (e.g., the pitcher positioned to be pouring away from the glass). This behavioral benefit, which we term the scene-

\footnotetext{
Received Dec. 9, 2010; revised March 21, 2011; accepted May 2, 2011

Author contributions: J.G.K., I.B., and C.-H.J. designed research; J.G.K. performed research; J.G.K. analyzed data; J.G.K., I.B., and C.-H.J. wrote the paper.

This work was supported by National Science Foundation (NSF) Grant 10-15645 to J.G.K.; NSF Grants 04-20794, 0531177, and 06-17699 to I.B.; and National Science Council (NSC) Grants 97-2511-S-008-005-MY3, 99-2410-H-008-022MY3, and 98-2517-S-004-001-MY3 to C.-H.J.C.-H.J. was supported by the NSC, Taiwan Grant 98-2918-1-008-011, and the Fulbright scholarship. We thank Neil Muggleton for his helpful discussion about the work and Jiaxin Yu for his assistance throughout the data collection process.

The authors declare no competing financial interests.

Correspondence should be addressed to any of the following: Jiye G. Kim, Department of Psychology, University of Southern California, 3620 McClintock Avenue, Los Angeles, CA 90089, E-mail: jiyekim@usc.edu; Dr. Irving Biederman, Neuroscience Program, University of Southern California, 3641 Watt Way, Los Angeles, CA 90089, E-mail: bieder@usc.edu; or Dr. Chi-Hung Juan, Institute of Cognitive Neuroscience, National Central University, Jhongli 320, Taiwan, E-mail: chijuan@cc.ncu.edu.tw.

DOI:10.1523/JNEUROSCI.6450-10.2011

Copyright $\odot 2011$ the authors $\quad 0270-6474 / 11 / 318320-05 \$ 15.00 / 0$
}

facilitation effect, has been shown in other behavioral tasks including visual search (Green and Hummel, 2004) and cued recall (Epstein et al., 1960).

Recent fMRI studies have shown that pairs of objects depicted as interacting elicit greater activity than noninteracting pairs in the lateral occipital cortex (LO) (Kim and Biederman, 2010; Roberts and Humphreys, 2010), a region critical for shape-based object recognition (Malach et al., 1995; James et al., 2003), rendering LO a potential candidate for the locus of the origin of the scene-facilitation effect. However, the intraparietal sulcus (IPS) also showed (although less consistently) a pattern of responses similar to that of LO (Kim and Biederman, 2010), leaving open the possibility that activity in LO is dependent on IPS (or vice versa).

IPS has been shown to be sensitive to visual attentional demands (Wojciulik and Kanwisher, 1999; Kanwisher and Wojciulik, 2000), and this activity can, in turn, modulate activity in ventral visual areas (Kastner et al., 1999; Martínez et al., 1999). That IPS activity is specific to visual processing and not driven by any effortful task has been shown by Wojciulik and Kanwisher (1999). An overlapping region in IPS was active across tasks including peripheral shifting, object matching, and a nonspatial conjunction task, but it was not active when the same group of subjects engaged in a language task. To the extent that interacting objects could attract more attention (as such pairs may elicit more interpretation), it is possible that the previous fMRI studies 


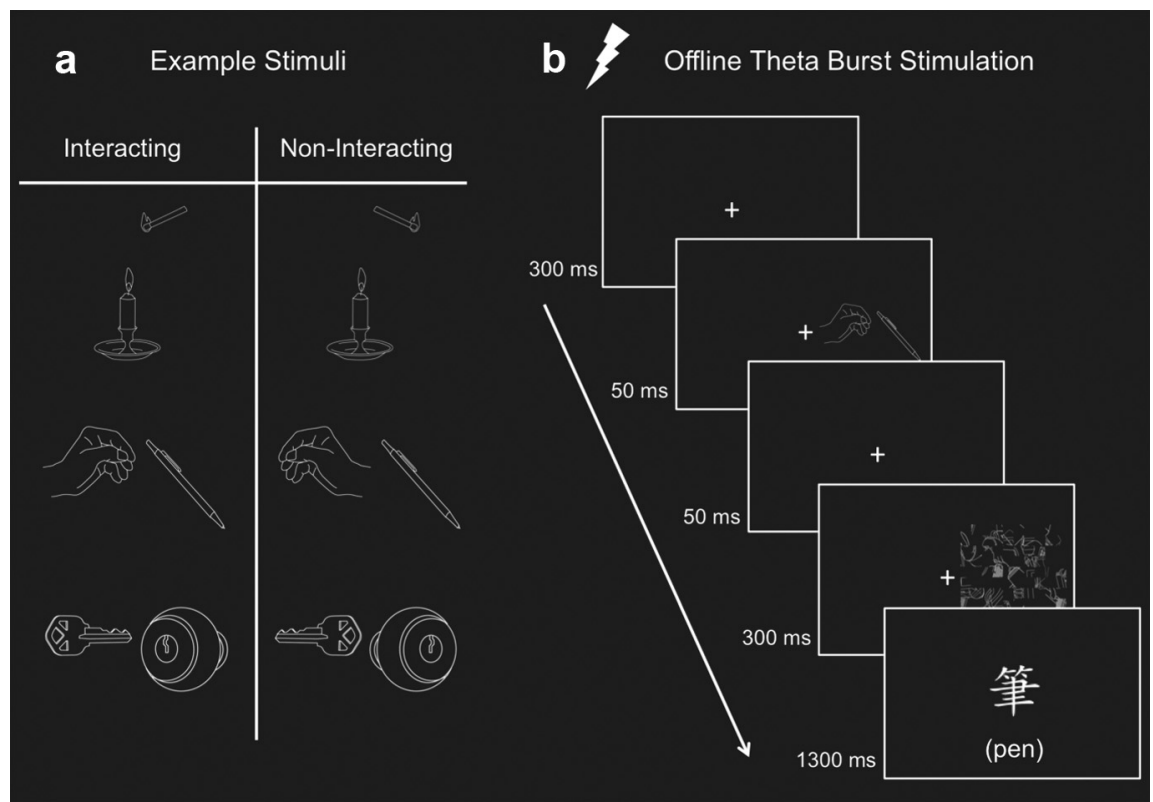

Figure 1. Sample stimuli and the presentation sequence for the target detection task. $\boldsymbol{a}$, Sample stimuli for the interacting and noninteracting conditions. $\boldsymbol{b}$, TMS was applied before the start of the $L$ and IPS sessions. An example of an RVF interacting trial is shown where the correct response is "present." In the experiment, the target label was shown only in Chinese characters.

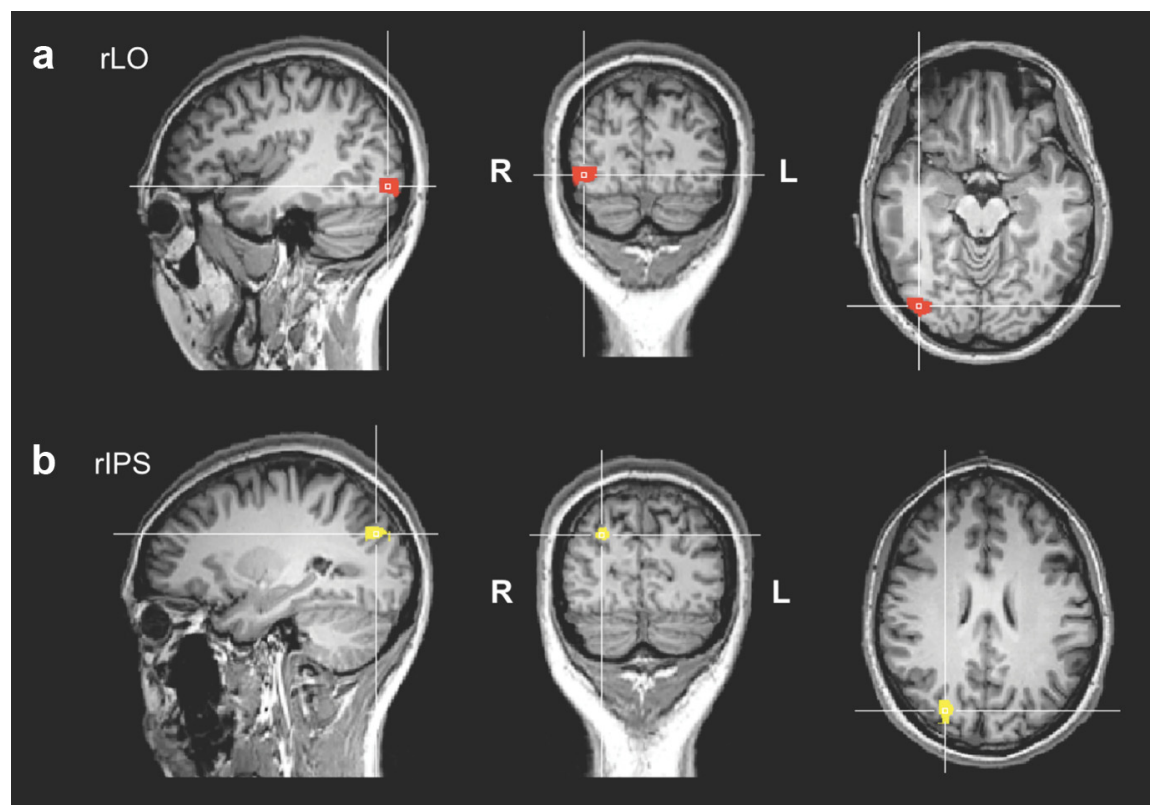

Figure 2. TMS stimulation sites. Functionally defined with fMRI localizer scans, rL0 $(\boldsymbol{a})$ and rIPS $(\boldsymbol{b})$ are shown on one representative subject's brain. The crosshairs indicate the center coordinates where TMS was delivered.

showing greater LO activity to interacting than noninteracting objects was dependent on IPS.

To determine whether LO and/or IPS might be critical for processing object interactions, we compared performance in a target detection task when offline theta burst repetitive transcranial magnetic stimulation (TMS) was delivered to the right $\mathrm{LO}(\mathrm{rLO})$ and right IPS (rIPS) and when TMS was not delivered. TMS was delivered only to the right hemisphere as lesions to the right hemisphere produce greater deficits in scene processing than lesions to the left (Milner, 1958). TMS administered to a particular region of interest (ROI) can disrupt the normal processing temporarily, allowing a test as to whether that region is essential for a specific cognitive process. If normal LO or IPS activity is required for producing the scene-facilitation effect, TMS delivered to LO or IPS would be expected to abolish that effect.

\section{Materials and Methods}

Subjects. Twelve subjects (nine men, mean age $=22.8$ years, range: $19-28$ years; all were right handed and all had normal or correctedto-normal vision) who were native Chinese speakers from the National Central University participated in the experiment. Participants received monetary compensation, were screened for safety, and gave informed consent in accordance with procedures approved by the local ethics committee.

Stimuli and procedures. Stimuli were selected from a set of 46 line drawings of individual objects. These were combined, pairwise, to make 23 different two-object interacting scenes. The noninteracting scenes were created by mirror reversing either one or both of the objects in each scene (Fig. 1a). Each object subtended an average of $2^{\circ} \times 2^{\circ}$ and the center of each scene was presented $4.5^{\circ}$ either to the left or right of central fixation.

Subjects performed the target detection task, modified from that of Green and Hummel (2006), where on each trial, two objects were depicted as either interacting (Inter) or not interacting (No-Inter). Subjects responded with button presses if a target label (written in Chinese characters) matched one of the two simultaneously presented objects (Fig. 1b). Critically, the target label was shown after the object pairs so as to not bias the subjects to look for the target object when the stimuli appeared. The objects were shown either in the left visual field (LVF), contralateral to the TMS site, or the right visual field (RVF), ipsilateral to the stimulation site, chosen randomly with equal probability. The object pairs were followed by a mask, which was created by randomly selecting four objects, rotating them $90^{\circ}$, and dividing each object into an $8 \times 8$ grid, whose cells were then shuffled and randomly selected for one of the 64 positions. Fifty percent of the trials were target-match trials. Participants were instructed to maintain central fixation and to respond as quickly and as accurately as possible.

Each participant completed three sessions (No-TMS, TMS to LO, and TMS to IPS) across 2 different days. The TMS sessions were separated by $1 \mathrm{~d}$ and the order of the sessions was counterbalanced across subjects. During each session, participants completed two blocks, each consisting of 368 trials, lasting $\sim 12$ min each. Before the first session, participants were given practice trials with 16 new objects not included in the main experiment.

To confirm that target detection in our experimental setup would be more accurate when object pairs are shown as interacting compared with noninteracting, as had been reported by Green and Hummel (2006), we ran a preliminary study with 14 subjects that replicated their results with higher $d^{\prime}$ values for interacting (3.2) than noninteracting (2.9) depictions, $t_{(13)}=3.61, p=0.003$

fMRI parameters and ROI localization. Functional and anatomical MRI scans were performed for each subject to determine coordinates for the TMS sites using a Siemens MAGNETOM Trio 3T scanner with a 
12-channel coil at the MRI Research Center, National Yang-Ming University. One anatomical (T1-weighted scan with the MPRAGE sequence with $\mathrm{TR}=1950 \mathrm{~ms}$, TE $=2.26 \mathrm{~ms}, 1 \times 1 \times 1 \mathrm{~mm}$ voxels, and 160 sagittal slices) and two functional scans (using a $\mathrm{T} 2{ }^{\star}$-weighted echo planar sequence with $\mathrm{TR}=2000 \mathrm{~ms}, \mathrm{TE}=30 \mathrm{~ms}, \mathrm{FOV}=192$, flip angle $=90^{\circ}$, voxel size $=3 \times 3 \times 3 \mathrm{~mm}$, and 33 transversal slices) were run for each subject.

Functional localizer runs were composed of sixteen $12 \mathrm{~s}$ blocks with alternating blocks between intact objects, places, faces, and scrambled images. Each image subtended a visual angle of $6^{\circ} \times 6^{\circ}$ presented centrally. Subjects were asked to passively view the stimuli. For each subject, rLO (Fig. $2 a$ ) was defined by comparing the contrast of object minus scrambled with a $t$-map threshold of $p<0.05$, Bonferroni corrected in the dorsal-caudal region in the right occipitotemporal region. Right IPS (Fig. $2 b$ ) was similarly defined as those voxels with the same contrast but in the intraparietal region.

This method of defining IPS was similar to that used by Xu and colleagues (Xu and Chun, 2006; Xu, 2008). By defining IPS this way, we guaranteed that this is the part of the parietal cortex that is specifically sensitive to object processing. Indeed, almost identical IPS loci have been identified as being involved in different kinds of visual attention tasks (Wojciulik and Kanwisher, 1999). Given the spatial resolution of TMS, it is highly likely that the region of IPS that we were stimulating overlapped to a large extent with the region implicated in visual attention. Although we did not use an attention task to functionally localize IPS, it is likely that merely viewing the intact objects would engage visual attention to a greater extent than viewing scrambled meaningless images because it is impossible to refrain from identifying meaningful objects. This point was documented by Smith and Magee (1980), who showed that when required to classify a word, "shirt," for example, as an article of clothing, subjects evidenced marked Stroop-like interference when the word was presented against a picture from another category (e.g., a sofa).

The fMRI scans were preprocessed and analyzed using BrainVoyager (Brain Innovation). All functional images were coregistered to each individual subject's anatomical scan. The anatomical scans were transformed into Talairach coordinates (Talairach and Tournoux, 1988), on which the statistical contrasts were performed to define the ROIs. Peak activation coordinates for $\mathrm{rLO}$ and rIPS were transformed back into each individual subject's native space using FSL software (FMRIB). Before each TMS session, each participant's TMS ROI site was coregistered to the anatomical MRI scan using the Brainsight system (Rogue Research) and the Polaris infrared tracking system (Northern Digital).

The mean peak Talairach coordinates across subjects for $\operatorname{rLO}(39.8$, $-71.5,-6.5)$ and rIPS $(26.8,-76.9,29.8)$ were comparable to previously reported coordinates (Xu and Chun, 2006; Kim et al., 2009; Kim and Biederman, 2010).

TMS and data analysis. A Magstim Super Rapid Stimulator was used to deliver TMS pulses to LO and IPS using a figure-of-eight coil with a diameter of $70 \mathrm{~mm}$. A theta burst stimulation protocol was the same as that used in the study by Vallesi et al. (2007) with the following parameters: three pulses given at $50 \mathrm{~Hz}$ every $200 \mathrm{~ms}$ (at $0 \mathrm{~ms}, 20 \mathrm{~ms}$, and $40 \mathrm{~ms}$ followed by $160 \mathrm{~ms}$ of rest) for $20 \mathrm{~s}$. This resulted in a total of 300 pulses per session, with the effect of TMS expected to last $\sim 20-30 \mathrm{~min}$ (Huang et al., 2005; Nyffeler et al., 2006). A single threshold of $40 \%$ of the maximal stimulator output ( 2 tesla) was used based on past studies showing reliable TMS effects across a wide range of tasks (Liu et al., 2010; Chao et al., 2011) and because motor cortex excitability does not provide a good guide to TMS thresholds in other cortical regions (Stewart et al., 2001). The coil handle was placed at each ROI pointed upward and parallel to the midsagittal plane.

A repeated measures $3 \times 2 \times 2$ ANOVA with TMS site (No-TMS, LO, and IPS), visual field (LVF vs RVF), and relation (Inter vs No-Inter) was run with the $d^{\prime}$ and reaction time (RT) data.

\section{Results}

There was no overall difference in performance to the target detection task across the different stimulation conditions, $F_{(2,10)}=$ $0.74, p=0.5$. Consistent with previous findings (Green and Hummel, 2006, and the pilot results), subjects were more accu-
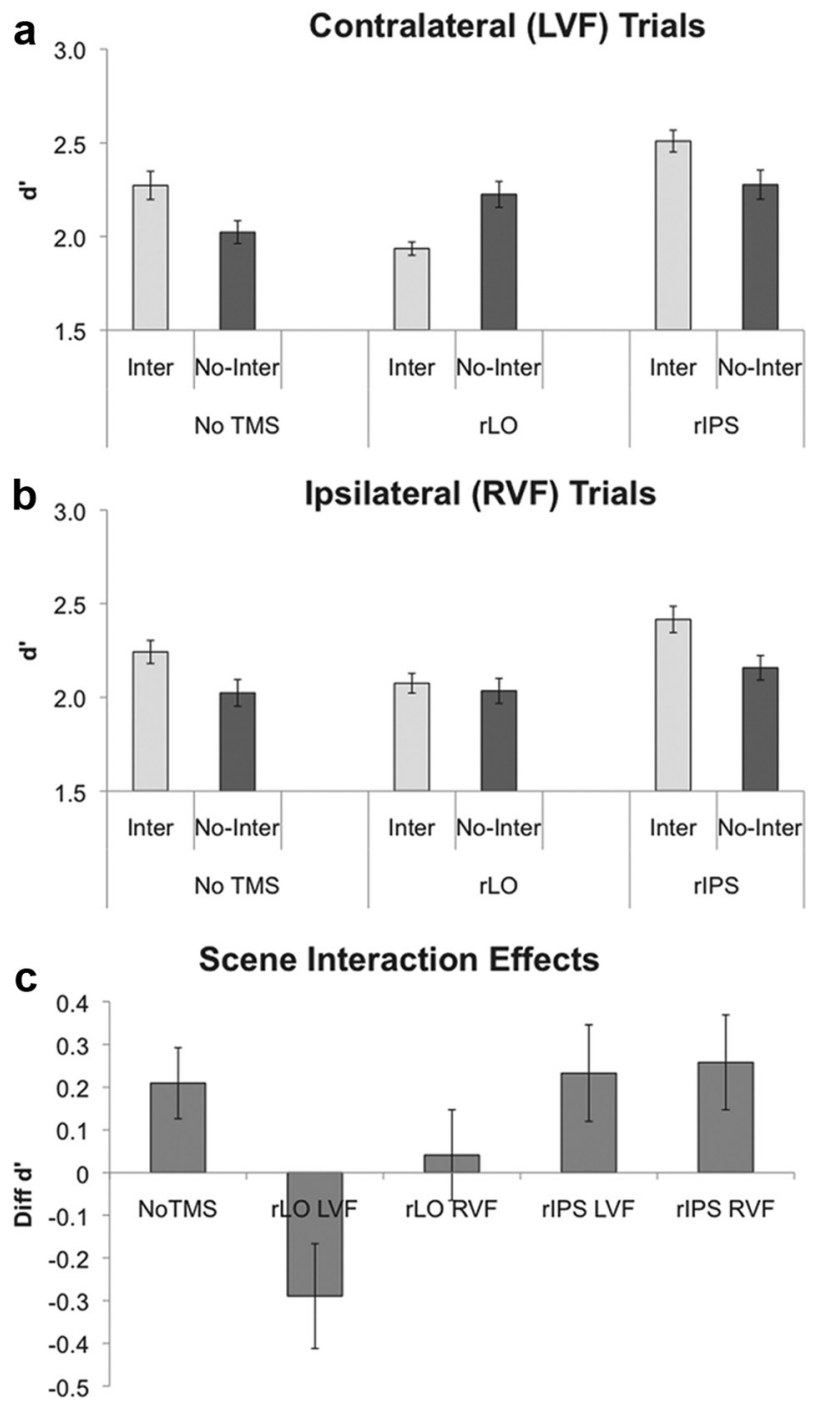

Figure 3. Accuracy for the target detection task. Accuracy (measured in $d^{\prime}$ ) for the contralateral $(\boldsymbol{a})$ and ipsilateral $(\boldsymbol{b})$ trials are shown. $\boldsymbol{c}$, Scene-facilitation effect $\left(d^{\prime}\right.$ for the Inter minus No-Inter conditions) is shown. The scene-facilitation effect of the No-TMS condition is collapsed across the LVF and RVF trials. Critically, the positive scene-facilitation effect was abolished after TMS was delivered to $\mathrm{LO}$ compared with the No-TMS or IPS sessions. All error bars represent the SEs computed from the deviation scores around each subject's overall $d^{\prime}$ for each session.

rate (higher $d^{\prime}$ values) when object pairs were depicted as interacting than noninteracting, showing a positive scene-facilitation effect, $F_{(1,11)}=5.86, p=0.03$ (Fig. 3). Across both visual fields, the accuracy of the interacting and noninteracting conditions depended on the TMS site, producing a two-way interaction of TMS site and relation, $F_{(2,10)}=11.94, p=0.002$. The amount of the scene-facilitation effect varied across TMS site and visual field, producing a significant three-way interaction, $F_{(2,10)}=$ 4.57, $p=0.04$. The interaction of TMS site and visual field was not significant, $F_{(2,10)}=0.20, p=0.82$.

A within-subjects contrast analysis revealed that the magnitude of the scene-facilitation effect was significantly reduced when TMS was delivered to LO $\left(\Delta d^{\prime}=0.18\right)$ compared with the No-TMS $\left(\Delta d^{\prime}=0.23\right)$ condition, $F_{(1,11)}=14.15, p<0.01$. The scene-facilitation effect did not differ between No-TMS and IPS sessions $\left(\Delta d^{\prime}=0.25\right), F_{(1,11)}<1.00$. Whereas the magnitude of the scene-facilitation effects did not differ across visual fields in the No-TMS session $\left(\Delta d^{\prime}=0.25\right.$ for LVF and $\Delta d^{\prime}=0.22$ for RVF), it was significantly lower for the LVF trials $\left(\Delta d^{\prime}=-0.41\right)$ 
than for the RVF trials $\left(\Delta d^{\prime}=0.04\right)$, when TMS was delivered to LO, $F_{(1,11)}=9.60, p=0.01$. The comparison between the magnitudes of the differences of the scene-facilitation effects across visual fields for IPS ( $\Delta d^{\prime}=0.23$ for LVF and $\Delta d^{\prime}=0.26$ for RVF) and No-TMS was not significant, $F_{(1,11)}<1.00$.

Critically, the positive scene-facilitation effect observed in the baseline condition was maintained when TMS was delivered to IPS but was completely lost when TMS was delivered to LO.

Although the mean accuracy after TMS was delivered to IPS was higher than that of the No-TMS condition, these differences were not close to reaching significance and thus likely reflect noise. The statistical comparison for the LVF trials between the Inter No-TMS and Inter IPS conditions was $t_{(11)}<1.00, p=0.46$; that between the LVF trials for the No-Inter No-TMS and NoInter IPS conditions was $t_{(11)}<1.00, p=0.41$. Because the NoTMS, LO, and IPS conditions were divided into three sessions across 2 different days, effects of practice and sessions likely contributed to the variability of performance across these conditions but not to the Inter and No-Inter comparisons, which were run in the same session.

The differences in RTs across conditions were very small, but subjects were reliably faster for the interacting $(859 \mathrm{~ms})$ than the noninteracting (864 ms) conditions, $F_{(1,11)}=9.97, p=0.009$. RT was also marginally faster for the LVF $(859 \mathrm{~ms})$ than RVF trials (865 ms), $F_{(1,11)}=4.61, p=0.06$. There were no other reliable effects on RT $(0.30<p$ values $<0.76)$.

\section{Discussion}

In the absence of TMS, accuracy of the target detection task was greater when the object pairs were shown as interacting than noninteracting. The benefit of interactions, which previously has been shown to depend on the grouping of the two objects into a single percept (Green and Hummel, 2004, 2006; Riddoch et al., 2010), is consistent with various behavioral studies demonstrating that object recognition is subject to contextual influences (Biederman, 1972; Palmer, 1975; Biederman et al., 1982). The application of TMS to LO, but not IPS, abolished the benefit of the interactions, thus demonstrating that LO, and not IPS, is a critical region for the coding of object interactions.

The coding of object relations in LO, and not IPS, is consistent with studies with parietal lobe patients who manifest extinction. Whereas these patients show failure to report one of two simultaneously presented objects, they show significant recovery of extinction when the two objects are depicted as interacting with each other (Riddoch et al., 2003). The recovery from extinction depends on the functional relationship between the objects (e.g., a pencil positioned toward a ruler to draw a line) and not the semantic associations or the statistical regularities in which they tend to co-occur in real life (e.g., a pencil and a pen). It is possible that an intact LO allows organization of the two objects into one perceptual unit by encoding the spatial relations between the two objects.

The direction of the scene-facilitation effect for the contralateral trials after TMS was delivered to LO was opposite (negative) to that of the No-TMS condition, meaning that not only was the facilitation from the interactions lost but there was a decrement of performance when object pairs were shown as interacting compared with when they were depicted as noninteracting. One, admittedly speculative, account of this effect is that the relations in the Inter condition presented familiar patterns between objects that invited interpretations that could be readily achieved in the absence of TMS. These interpretations could have facilitated object identification. With TMS delivered to LO, however, subjects might have experienced greater difficulty in achieving such interpretations with a resultant cost in their capacity for identifying the individual objects. Such interpretive difficulty is suggested by the finding by Milner (1958) that right temporal lesions reduced the ability of subjects to detect scene anomalies.

TMS delivered to LO had a larger effect on scenes presented to the contralateral than the ipsilateral visual field, an effect consistent with the greater representation of objects presented in the contralateral than ipsilateral visual fields in that area (GrillSpector et al., 1998; Tootell et al., 1998).

IPS is generally considered to be part of an attentional network that is selectively sensitive to shape responses (Wojciulik and Kanwisher, 1999; Denys et al., 2004), which can selectively modulate responses in ventral visual areas associated with enhanced processing (Kastner et al., 1999; Martínez et al., 1999). TMS delivered to IPS did not change the behavioral benefit of coding of object interactions. Whatever general attentional effect IPS has on LO, it does not seem to affect the processing coding of interactions between objects.

Activity in IPS has also been implicated in visuomotor responses to static objects that have implied action (Sakata et al., 1995; Grèzes and Decety, 2002). To the extent that interacting objects afford more effective action than noninteracting objects, action affordances could be the source of the scene-facilitation effect. Our results suggest that should action affordance be the source, it is not driven by IPS activity. Because IPS is likely a subregion of a potentially large number of areas involved in processing visual action affordances (Riddoch et al., 2003; Culham and Valyear, 2006), future investigation of other regions is warranted to assess their possible roles in the processing of action relatedness.

LO is the earliest region in the ventral pathway where intact shapes are distinguished from their scrambled counterparts (Malach et al., 1995), and it is critical for shape-based object recognition (James et al., 2003). TMS delivered to LO previously has been shown to disrupt processes associated with recognition of individual objects as assessed by object naming and identity matching (Chouinard et al., 2009; Pitcher et al., 2009), but little is known about its functioning with multiple objects. Whereas the scene-facilitation effect was invariant to TMS when delivered to IPS, TMS delivered to LO completely abolished the benefit of scene interactions. This study thus provides strong evidence for LO's critical role in the processing of such relations, consistent with fMRI studies showing sensitivity to object interactions in LO (Kim and Biederman, 2010; Roberts and Humphreys, 2010). The coding of interobject relations is thus not relegated to an attentional cortical region, such as IPS, but occurs at the same cortical locus and likely is simultaneous with the processing of object shape.

\section{References}

Biederman I (1972) Perceiving real-world scenes. Science 177:77-80.

Biederman I, Rabinowitz JC, Glass AL, Stacy EW Jr (1974) On the information extracted from a glance at a scene. J Exp Psychol 103:597-600.

Biederman I, Mezzanotte RJ, Rabinowitz JC (1982) Scene perception: detecting and judging objects undergoing relational violations. Cogn Psychol 14:143-177.

Chao CM, Tseng P, Hsu TY, Su JH, Tzeng OJ, Hung DL, Muggleton NG, Juan $\mathrm{CH}$ (2011) Predictability of saccadic behaviors and the human posterior parietal cortex. Hum Brain Mapp. Advance online publication. Retrieved Dec. 10, 2010. doi: 10.1002/hbm.21162.

Chouinard PA, Whitwell RL, Goodale MA (2009) The lateral-occipital and the inferior-frontal cortex play different roles during the naming of visually presented objects. Hum Brain Mapp 30:3851-3864. 
Culham JC, Valyear KF (2006) Human parietal cortex in action. Curr Opin Neurobiol 16:205-212.

Denys K, Vanduffel W, Fize D, Nelissen K, Peuskens H, Van Essen D, Orban GA (2004) The processing of visual shape in the cerebral cortex of human and nonhuman primates: a functional magnetic resonance imaging study. J Neurosci 24:2551-2565.

Epstein W, Rock I, Zuckerman CB (1960) Meaning and familiarity in associative learning. Psychol Monog Gen Appl 74:1-22.

Green C, Hummel JE (2004) Functional interactions affect object detection in non-scene displays. In: Proceedings of the $26^{\text {th }}$ Annual Meeting of the Cognitive Science Society (Forbus K, Gentner D, Reiger T, eds), pp $488-$ 493. Mahwah, NJ: Erlbaum.

Green C, Hummel JE (2006) Familiar interacting object pairs are perceptually grouped. J Exp Psychol Hum Percept Perform 32:1107-1119.

Grèzes J, Decety J (2002) Does visual perception of object afford action? Evidence from a neuroimaging study. Neuropsychologia 40:212-222.

Grill-Spector K, Kushnir T, Hendler T, Edelman S, Itzchak Y, Malach R (1998) A sequence of object-processing stages revealed by fMRI in the human occipital lobe. Hum Brain Mapp 6:316-328.

Huang YZ, Edwards MJ, Rounis E, Bhatia KP, Rothwell JC (2005) Theta burst stimulation of the human motor cortex. Neuron 45:201-206.

James TW, Culham J, Humphrey GK, Milner AD, Goodale MA (2003) Ventral occipital lesions impair object recognition but not object-directed grasping: an fMRI study. Brain 126:2463-2475.

Kanwisher N, Wojciulik E (2000) Visual attention: insights from brain imaging. Nat Rev Neurosci 1:91-100.

Kastner S, Pinsk MA, De Weerd P, Desimone R, Ungerleider LG (1999) Increased activity in human visual cortex during directed attention in the absence of visual stimulation. Neuron 22:751-761.

Kim JG, Biederman I (2010) Where do objects become scenes? Cereb Cortex. Advance online publication. Retrieved Dec. 8, 2010. doi: 10.1093/cercor/bhq240.

Kim JG, Biederman I, Lescroart MD, Hayworth KJ (2009) Adaptation to objects in the lateral occipital complex (LOC): shape or semantics? Vision Res 49:2297-2305.

Liu CL, Tseng P, Chiau HY, Liang WK, Hung DL, Tzeng OJL, Muggleton NG, Juan CH (2010) The location probability effects of saccade reaction times are modulated in the frontal eye fields but not in the supplementary eye field. Cereb Cortex. Advance online publication. Retrieved Dec. 10, 2010. doi: 10.1093/cercor/bhq222.

Malach R, Reppas JB, Benson RR, Kwong KK, Jiang H, Kennedy WA, Ledden PJ, Brady TJ, Rosen BR, Tootell RB (1995) Object-related activity revealed by functional magnetic resonance imaging in human occipital cortex. Proc Natl Acad Sci U S A 92:8135-8139.

Martínez A, Anllo-Vento L, Sereno MI, Frank LR, Buxton RB, Dubowitz DJ,
Wong EC, Hinrichs H, Heinze HJ, Hillyard SA (1999) Involvement of striate and extrastriate visual cortical areas in spatial attention. Nat Neurosci 2:364-369.

Milner B (1958) Psychological deficits produced by temporal-lobe excision. Proc Assoc Res Nerv Mental Dis 36:244-257.

Nyffeler T, Wurtz P, Lüscher HR, Hess CW, Senn W, Pflugshaupt T, von Wartburg R, Lüthi M, Müri RM (2006) Repetitive TMS over the human oculomotor cortex: comparison of $1 \mathrm{~Hz}$ and theta burst stimulation. Neurosci Lett 409:57-60.

Palmer SE (1975) The effects of contextual scenes on the identification of objects. Mem Cognit 3:519-526.

Pitcher D, Charles L, Devlin JT, Walsh V, Duchaine B (2009) Triple dissociation of faces, bodies, and object in extrastriate cortex. Curr Biol 19:319-324.

Riddoch MJ, Humphreys GW, Edwards S, Baker T, Willson K (2003) Seeing the action: neuropsychological evidence for action-based effects on object selection. Nat Neurosci 6:82-89.

Riddoch MJ, Bodley Scott S, Humphreys GW (2010) No direction home: extinction is affected by implicit motion. Cortex 46:678-684.

Roberts KL, Humphreys GW (2010) Action relationships concatenate representations of separate objects in the ventral visual system. Neuroimage 52:1541-1548.

Sakata H, Taira M, Murata A, Mine S (1995) Neural mechanisms of visual guidance of hand action in the parietal cortex of the monkey. Cereb Cortex 5:429-438.

Smith MC, Magee LE (1980) Tracing the time course of picture-word processing. J Exp Psychol Gen 109:373-392.

Stewart LM, Walsh V, Rothwell JC (2001) Motor and phosphene thresholds: a transcranial magnetic stimulation correlation study. Neuropsychologia 39:415-419.

Talairach J, Tournoux P (1988) Co-planar stereotaxic atlas of the human brain. Stuttgart, Germany: Thieme.

Tootell RB, Mendola JD, Hadjikhani NK, Liu AK, Dale AM (1998) The representation of the ipsilateral visual field in human cerebral cortex. Proc Natl Acad Sci U S A 95:818-824

Vallesi A, Shallice T, Walsh V (2007) Role of the prefrontal cortex in the foreperiod effect: TMS evidence for dual mechanisms in temporal preparation. Cereb Cortex 17:466-474.

Wojciulik E, Kanwisher N (1999) The generality of parietal involvement in visual attention. Neuron 23:747-764.

Xu Y (2008) Representing connected and disconnected shapes in human inferior intraparietal sulcus. Neuroimage 40:1849-1856.

Xu Y, Chun MM (2006) Dissociable neural mechanisms supporting visual short-term memory for objects. Nature 440:91-95. 